\title{
Shopping trip mode choice of older adults: an application of activity space and hybrid choice models in understanding the effects of built environment and personal goals
}

\author{
Samira Ramezani ${ }^{1}$ (D) Tiina Laatikainen $^{1}$ (D) Kamyar Hasanzadeh $^{1}$ (D) . \\ Marketta Kyttä ${ }^{1}$ (D)
}

Published online: 2 November 2019

(C) The Author(s) 2019

\begin{abstract}
Rapid growth of the older population worldwide, coupled with their overreliance on automobile and its negative consequences for the environment and for their wellbeing, has encouraged research on travel behavior of this age group. This study contributes to the literature by providing an integrated analysis of the effects of sociodemographic, built environmental, psycho-social, trip, and activity space attributes on shopping trip mode choice of older adults in Helsinki Metropolitan Area. Data was collected using an online map-based survey. Two person-based activity space models were developed, in addition to the commonly used 500-m buffer, to measure activity space and built environmental attributes. Integrated Choice and Latent Variable (ICLV) models were utilized to explore modal choice. Although the use of activity space models did not significantly increase the fit of ICLV models, it provided different information. Walkability index showed a positive significant effect on walking trips in individualized residential exposure model. A positive effect on transit use or biking was found in individual home range and 500-m buffer. The shape and dispersion of activity spaces affected mode choice as well. Green space influenced the goal of being physically active which in turn affected mode choice. Three personal goals of being physically active, having cultural and social affairs, and caring for others influenced mode choice. Results indicate the priority of the use of activity space and hybrid choice models in understanding travel behavior. Findings of this study can guide policies aiming to increase the use of more sustainable modes among this age group.
\end{abstract}

Keywords Modal choice $\cdot$ Activity space models $\cdot$ Hybrid choice models $\cdot$ Old adults Built environment $\cdot$ Personal goals

Samira Ramezani

samira.ramezani@aalto.fi

1 Department of Built Environment, Aalto University, Espoo, Finland 


\section{Introduction}

\section{Motivation}

With fast growth of older adults in Europe (European Commission 2014) and worldwide (United Nations 2002), research on travel behavior of this age group has increased in the last couple of decades. It is well documented in the literature that aging has significant consequences for the transport sector (e.g. Hjorthol et al. 2010; Mercado et al. 2007; Coughlin and D'Ambrosio 2012). Older adults constitute the fastest growing segment of the driving population, both in terms of license rates and kilometers travelled (Nakanishi and Black 2015; Böcker et al. 2017). This overreliance on automobile has negative impacts on the society not only in terms of the resulting environmental pollution and congestion (Banister and Bowling 2004), but also the effects on health and wellbeing of this vulnerable age group (e.g. Oxley and Whelan 2008; Kemperman and Timmermans 2014; Böcker et al. 2017). Substantial policy movements to make these societal challenges recognized in the national agenda have occurred in Europe and North America (Mercado et al. 2007). Consequently, research on travel behavior of the older population has grown to inform policy (Mercado et al. 2007; Böcker et al. 2017). Although progress has been made in understanding different sets of factors (e.g. travel factors, sociodemographic, built environmental, and latent psycho-social factors) affecting this age group's travel behavior and more specifically their mode choice, a number of conceptual and methodological shortcomings still exist in the literature.

\section{Research gaps}

First, while a plethora of studies have investigated the socio-demographic, built environmental, and travel factors influencing this age group's travel behavior and transport mode choices (e.g. Schwanen et al. 2001; Banister and Bowling 2004; Kim and Ulfarsson 2004; Páez et al. 2007; Schmöker et al. 2008; Hjorthol et al. 2010; Pettersson and Schmöcker 2010; Kim 2011; Van den Berg et al. 2011; Boschmann and Brady 2013; Hahn et al. 2016; Böcker et al. 2017; Liu et al. 2017), the heterogeneous travel behavior of this age group and the latent psycho-social factors affecting their travel behavior have rarely been accounted for. The studied travel factors mainly include travel time and cost (e.g. Su et al. 2009), and purpose of the trip (e.g. Schwanen et al. 2001; Schmöker et al. 2008; Su et al. 2009; Hjorthol et al. 2010). Particularly, inclusion of purpose of the trip in models and separately modelling different travel purposes has been emphasized in the literature. It has been well established that shopping is the main daily trip purpose among this age group (e.g. Schmöker et al. 2008; Su et al. 2009; Hjorthol et al. 2010), and that the older adults are more likely to use car when going shopping. After shopping there is generally a load to carry, which increases the difficulties to use other modes of transportation (e.g. Su et al. 2009). However, shopping trip mode choices are diverse and while car driving is, in general, found to be the most frequently used mode (e.g. Gould et al. 1998), some shopping trips are made by foot, transit, or bicycle (e.g. Su et al. 2009). In addition, when studying travel behavior of this age group, it is important to distinguish the "younger old" (e.g. 55-75) from the "older old" (above 75) as declines in health often limit feasible activities (which can include the use of active travel modes) for the older olds (e.g. Alsnih and Hensher 2003). Moreover, individuals may have heterogeneous travel behavior and choose 
different modes of transportation for shopping purpose in different days and for reaching different shopping locations. It is, therefore, of utmost importance to account for the heterogeneous travel behavior of this age group when investigating the factors that affect their shopping trip mode choice.

Besides travel factors, sociodemographic and built environmental factors affecting this age groups' mode choice have been widely investigated. The studied sociodemographic factors in the literature mainly include gender, age, education, employment status, income, and household size (Shao et al. 2019). The effects of physical health, size of social networks (e.g. Haustein 2012), and car ownership (Chen et al. 2004; Hjorthol et al. 2010) have been also investigated in a number of studies. The studied built environmental factors mainly include population density, land use, recreation and green areas, and walking distance/time to the public transport stops (Shao et al. 2019). Neighborhood accessibility measures (e.g. Haustein 2012) and, more recently, environmental factors, such as weather conditions and seasonality (e.g. Böcker et al. 2017), have been investigated as well (e.g. Böcker et al. 2017). However, there has been some contrasting results in the literature regarding the influence of built environmental factors on mode choice of this age group (see Böcker et al. 2017 for a review). Controversies regarding the influence of built environmental factors on transportation mode choice, regardless of age or trip purpose, have been widely discussed in the literature (see Ewing and Cervero 2010 for a review). However, most of the studies have shown positive correlations between the choice of active and sustainable modes of transportation and intersection density, land use mix and residential and commercial density which has been incorporated into a walkability index developed by Frank et al. (2010). This measure is reported to have had consistent significant positive correlations with the use of active modes of transportation for different age groups (e.g. Clark and Scott 2014). However, the possible effect of walkability index on shopping trip mode choice of older adults has not been investigated. In addition, while there is a consensus regarding the positive correlation between the percentage of green space area within older adults' neighborhood boundary and active travel or physical activity (e.g. Sallis et al. 2016; Lu 2018), the possible effect of this built environmental measure on shopping trip mode choice of this age group has been rarely studied.

While built environmental, sociodemographic, and travel factors have been investigated in a plethora of studies, very few studies have accounted for or controlled the effect of latent psycho-social factors such as the diverse lifestyles and attitudes of the older adults (e.g. Haustein 2012), as well as their self-reported physical and functional abilities (e.g. Naumann et al. 2009). Put differently, integrated studies that account for the full set of travel factors, socio-demographics, psycho-social, and built environmental factors influencing transportation mode choice of older adults at the same time are limited in the literature. Furthermore, more recently, a number of studies (e.g. Temme et al. 2008; Kamargianni et al. 2015) have emphasized the importance of investigating the possible effects of basic underlying constructs, such as one's personal goals, defined as states that people strive to achieve or avoid in the future (Freund and Riediger 2006; Saajanaho et al. 2016; SalmelaAro 2009), rather than using relatively superficial constructs such as the willingness or propensities to use specific modes of transportation (e.g. Haustein 2012), in order to gain a better understanding of travel behavior. For example, Temme et al. (2008) found that some abstract motivations such as hedonism and power influence transportation mode choice. Saajanaho et al. (2015) found that the older adults' spatial extent of mobility in daily life (defined as Life-space mobility) is influenced by personal goals. For example, older adults who had reported personal goals such as maintaining functioning, staying mentally alert, activeness in daily life, and meeting other people, had larger life-space mobility compared 
to those not reporting such goals. Moreover, older adults who did not report any personal goals moved around outside their home surroundings less than those who reported at least one personal goal in any of the goal categories of the study (for details see Saajanaho et al. 2015). Having personal goals related to maintenance of functioning as well as engaging in physical and social activities can influence not only the older adults' spatial extent of mobility but also the mode of transportation that they use due to the need to move around outside their home surroundings. However, such understandings of the possible influence of latent personal goals on transportation mode choice of older adults in general, and their shopping trip mode choice in particular are missing in the existing literature.

Second, the latent psycho-social variables, if included at all, have been always dealt with as other observable variables (i.e. the factor scores are first extracted using factor analysis of a set of Likert scale statements, and these factor scores are used subsequently as a continuous variable in models). This procedure might induce measurement error as the extracted values differ from the true values (see Bielaire 2010). Moreover, socio-demographic and built environmental factors may affect latent factors (Ramezani et al. 2015, 2018a, b) which in turn influence mode choice and such complexities cannot be accounted for and unraveled in commonly used modelling frameworks such as multinomial logit models. However, as Vij and Walker (2016, p. 205) put it, more recently, hybrid choice models (i.e. Integrated Choice and Latent Variable (ICLV) models) have been conceived to enrich "existing representations of decision-making through the inclusion of latent sociological and psychological constructs". While discrete choice models usually use sociodemographic variables and random error components as proxies for such latent behavioral constructs, they are not able to provide any additional information to describe what these variables might be proxies for (Vij and Walker 2016). On the contrary, an ICLV model can give structure and meaning to underlying differences in behavior by helping identify additional parameters (e.g. observable variables such as sociodemographic and built environmental factors) associated with the latent explanatory variables, and test for the effect of these latent variables on observable choices (Vij and Walker 2016). To the authors' knowledge, application of such a modelling framework in studying transportation mode choice of older adults is missing in the literature.

Finally yet importantly, studies of the effects of the built environment on travel behavior of older adults (as well as other age groups) have often used predefined and static spatial units of analysis around an individual's home location (e.g. 500-1000-m buffers, grids, census blocks) to measure the built environmental factors (e.g. Böcker et al. 2017). However, an individual's travel can be influenced not only by the urban form characteristics of home location but also by the characteristics of the destination location (e.g. Ding et al. 2018) and the route from home to destination (e.g. Krizek 2003). Moreover, the spatial extent to which attributes are perceived by individuals can differ (Schirmer et al. 2014). As Mitra and Buliung (2012, p. 53) put it, previous research (Banerjee and Baer 1984; Horton and Reynolds 1971) has shown that "a household's perceived neighborhood boundary can largely be defined based on the action space within which the household members perform their daily activities, and consume goods and services". The validity of the use of commonly used buffers to account for the effect of built environment on individuals' travel behavior has, therefore, become controversial (e.g. Perchoux et al. 2013; Holliday et al. 2017; Laatikainen et al. 2018). Such predefined spatial units of analysis have been criticized for not being able to define the spatiotemporal extents of individuals' neighborhoods and spatial exposure (e.g. Mitra and Buliung 2012; Perchoux et al. 2013; Holliday et al. 2017; Laatikainen et al. 2018). As Hasanzadeh et al. (2017) put it, although the use of buffer has priority over the traditionally rigid neighborhood definitions (such as large traffic 
analysis zones or administrative boundaries), such definition of neighborhood is individual-centered, but not individual specific for the spatial extent of neighborhood is systematically defined in a uniform way for all individuals. Recent research has, therefore, taken steps forward by defining dynamic and person-based "activity space" as the spatial unit of analysis. These approaches are based on the understanding that the spatial units of analysis should not only be individual-based, but also individual specific (Hasanzadeh et al. 2017). Places and daily movements, or space in general, are heterogonous and complex in nature and this should be considered in the modeling of activity spaces (Wei et al. 2018). Therefore, few studies have proposed the use of advanced models of activity space which have been mostly used in health related studies (e.g. Wang et al. 2018; Wei et al. 2018) or for measuring hotspots of segregation and integration potentials in cities using patterns of daily travel behavior (e.g. Farber et al. 2015).

For example, in an attempt to study the spatial inequalities of the food environment ("foodscape") and measure their effects on personal health, Wei et al. (2018) used longterm GPS data to model individual activity spaces. The authors used a framework based on space-time proximity to investigate individual foodscape exposure. In a similar attempt, Wang et al. (2018) used a "crystal-growth" algorithm to develop an individual activity space model that not only considers people's actual daily activity patterns based on GPS tracks but also takes into account the environmental contexts constraining or encouraging people's daily activities. There is little doubt that such advanced methods provide a more accurate estimation of individual activity spaces compared to more simplistic approaches. However, a crucial fact that needs to be taken into consideration when developing activity space models is their application and ease of use. In response, Hasanzadeh et al. (2017, 2018) developed two individually and spatially sensitive models of activity spaces, which are also practical to use. The individual home range model (IHR) is an individual-specific boundary method, which applies a convex hull enclosing all daily destination points as well as the home point (Hasanzadeh et al. 2017). Individualized residential exposure model (IREM) is based on the understanding that the activity spaces are not only variable in their shape and extents, but also in their very fabric (Hasanzadeh et al. 2018). However, to the authors' knowledge, application of such activity space models in studying the effect of built environment on transportation mode choice of older adults is missing in the literature.

\section{Aim and scope of the study}

This study aims to contribute to the understanding of transportation mode choice of older adults (i.e. aged 55-75) by filling some of the abovementioned gaps. The contribution of this study is threefold. First, it uses an ICLV modelling framework to investigate the effects of different sets of variables including travel factors (e.g. travel time and cost), socio-demographics, psycho-social, and built environmental factors, influencing transportation mode choice of older adults for shopping trips as one of the main daily trip purposes among this age group (e.g. Schmöker et al. 2008; Su et al. 2009; Hjorthol et al. 2010). The aim is to investigate the observable socio-economic and built environmental factors influencing older adults' transportation mode choice directly, and through their influence on latent psycho-social factors. Put differently, such a modelling framework is used to enhance understanding of the behavior of heterogeneous groups of older adults by unraveling the association between the observable (e.g. sociodemographic, built environmental) and the latent (i.e. psycho-social) factors affecting mode choice. Such an understanding can guide policy making by providing information about target groups for different policies that aim to increase the use of more sustainable and 
active transportation modes. Moreover, by using panel data (more than one shopping trip, to one or several shopping locations, reported by each respondent), this study accounts for the heterogeneous travel behavior of the respondents in contrast to most of the previous studies that consider only one trip and one shopping location reported per respondent. In this study we use the definition of shopping trips by Barber (1995) and used by Su et al. (2009) who define a shopping trip as a trip to any retail center, irrespective of the size and type of the store or shop and of whether or not a purchase is made. Second, rather than examining the effects of commonly studied latent factors, such as willingness to use specific modes of transportation, the concept of personal goals, defined as states that people strive to achieve or avoid in the future (Freund and Riediger 2006; Saajanaho et al. 2016; Salmela-Aro 2009), is used to study the intrapersonal and psychological dimensions (Laatikainen et al. 2019) that could affect the older adults' transportation mode choice. Four personal goals, namely "being physically active and doing sports", "caring for others", "managing on one's own", and "engaging in cultural and social affairs" are examined. Last but not least, this study contributes to the literature by using dynamic person based spatial units of analysis (i.e. activity space models) in addition to the commonly used 500-m buffer in studying the effect of urban form factors on transportation mode choice. Two recently developed activity space modelling methods, individual home range (Hasanzadeh et al. 2017) and individualized residential exposure model (Hasanzadeh et al. 2018; Hasanzadeh 2018), are used to define person based spatial units of analysis based on respondents' revealed daily activities (Laatikainen et al. 2018). To the authors' knowledge, this is the first study that uses these activity space models in studying the effect of urban form factors on transportation mode choice of older adults. In addition, the possible effects of the shape and dispersion of an individual's activity space on their transportation mode choice is investigated. The aim is to examine whether the use of these different spatial units of analysis affects the results we obtain regarding how and to what degree the built environment affects transportation mode choice and whether the activity space models can provide us with better insights for policymaking.

To cap it all, this study aims to answer the following research questions:

- Do personal goals of older adults affect their shopping trip mode choice?

- To what degree and how do sociodemographic and built environmental factors affect shopping trip mode choice of older adults?

- Does the use of different spatial units of analysis (activity space models versus the commonly used 500-m buffer) affect the results we obtain regarding the effects of built environment?

- Does the shape of activity space and activity dispersion of older adults affect their transportation mode choice?

The paper is organized as follows. The next section ("Methodology" section) provides a detailed description of research methodology, including the data collection and data analysis procedure. "Results and discussion" section presents and discusses the results. Finally, the last section concludes the paper with a summary of key findings and future research directions. 


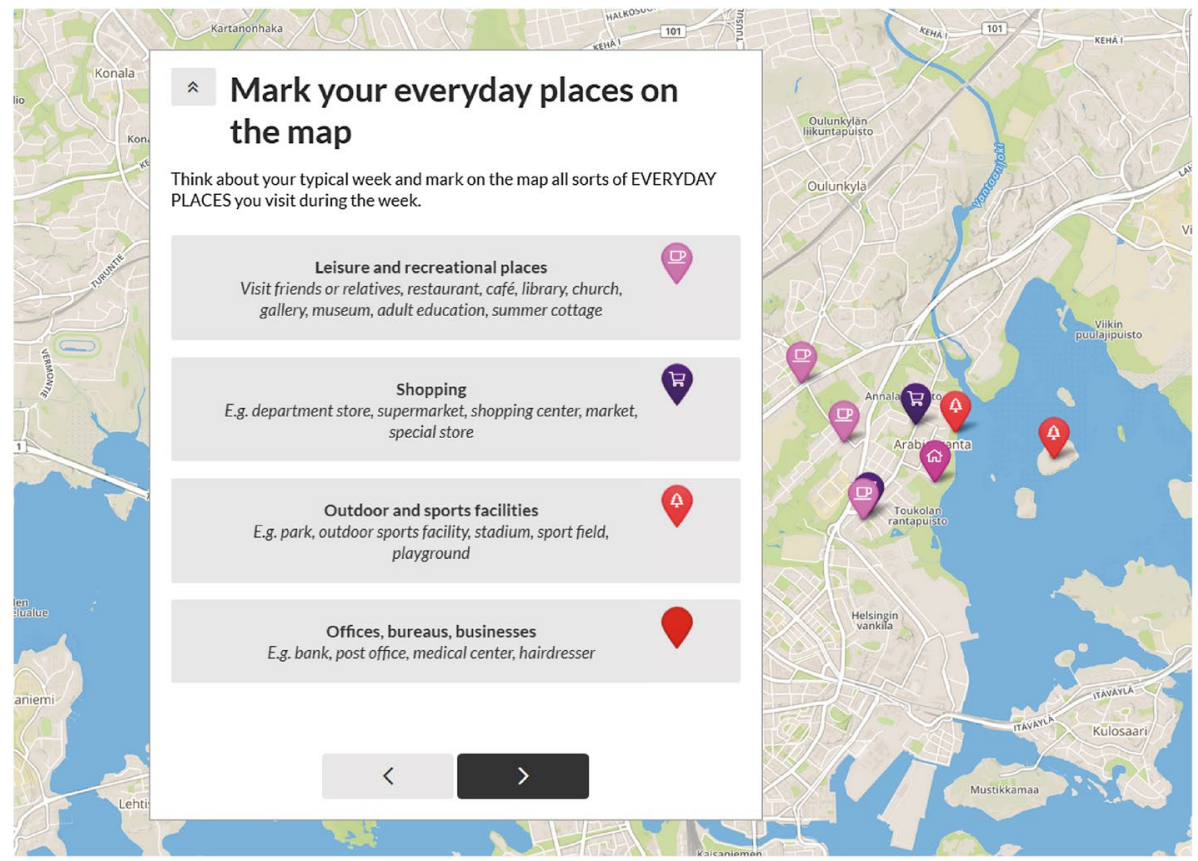

Fig. 1 The online interface of the survey where respondents marked on a map their everyday errand points in a typical week

\section{Methodology}

\section{Data collection procedure}

The data used in this study was collected using an online map-based survey tool, namely public participation GIS (PPGIS). In addition to commonly collected data, such as sociodemographic information, using traditional paper-based surveys or common online survey tools, this method allows for collection of place-based data with geographical coordinates, which can be easily visualized, and analyzed in Arc GIS. The survey was designed to collect the respondents' sociodemographic characteristics, their personal goals based on ratings of nineteen Likert-scale statements on a scale of 0 (=not important) to 6 (=very important), the locations they visit during a typical week, the modes of transport usually used to reach those places, and the frequency of visit per month. This study focuses on shopping trip mode choice. However, the respondents' markings of all types of everyday places (i.e., shopping place, leisure and recreational place, outdoor and sport facilities, and personal errands such as visiting post office and banks) they visited during a typical week, provided necessary information for modelling their personal activity space, which is described in more details in "Spatial units of analysis" section. Figure 1 shows the online interface used for collection of the place-based data. Details about the indicators of personal goals are provided in "Personal goals within the sample population" section.

Finland has one of the oldest populations in Europe (European Commission 2018). Helsinki, the capital of Finland, makes an interesting setting for studying older adults' transportation mode choice as the share between different transport modes is rather equal, 
excluding bicycling. In 2017, 35\% of all journeys done in Helsinki were by walking, 34\% by public transit, $31 \%$ by private car, but only $9 \%$ by cycling (Jaakola et al. 2018). Helsinki Metropolitan Area has a diverse retail structure. In the whole study area, shops for daily goods or grocery stores are well accessible by bike and/or on foot. In addition, shopping centers that accommodate a combination of department stores, hypermarkets, and individual retail shops (for products such as clothing, home appliances, cosmetics, etc.) are also available in the local neighborhood centers, which are accessible by bike and/or on foot. These shopping centers are usually located on or close to subway stations or are well accessible by other means of public transport as well (e.g. bus). However, some shopping centers are located outside the inner city alongside highways, which can be more easily accessed by car or by bus rather than on foot or by bike.

The survey targeted the older adults (55-75-year olds) living in Helsinki Metropolitan Area (HMA), Finland. The contact information of a random sample of 5000 residents of this age group was acquired from Finland's Population Register Center in 2015. An invitation letter to participate in the online survey was mailed to the sample. 1139 responses were received, 844 of which had provided complete answers. The data showed general consistency on most socio-demographic variables within the study region. For more information about the sociodemographic characteristics of the respondents and its comparison to statistics of the region's population, see earlier articles using this dataset (e.g. Hasanzadeh et al. 2018; Laatikainen et al. 2019).

Since this study focuses on shopping trips, and as this research accounts for the heterogeneous shopping trip behavior of the older adults, only those who had marked at least two shopping trips (which could be trips reported to one single shopping location or several shopping locations) were taken for further analysis, which provided us with a panel data and a sample size of 607 . This sample consisted of 360 women (204 of the female sample were 55-64 year-olds and 156 of them were 65-75 year olds) and 247 men (139 were 55-64 year-olds and 108 were 65-75 year-olds).

\section{Data analysis}

Data analysis was conducted in four stages. First, the three spatial units of analysis (i.e. the 500-m buffer, individual home range, and individualized residential exposure models) were modelled using ArcGIS 10.6 ("Spatial units of analysis" section). These spatial units of analysis were used to measure the built environmental factors ("Built environmental measures" section). Factor analysis was then conducted with the ratings of the Likert-scale statements to identify the personal goals within the sample population ("Personal goals within the sample population" section). The last stage of analysis was the mode choice modelling which explored the effect of different factors on modal choice. The built environmental factors and the personal goals found in the former stages were used in the process of model development ("Mode choice modelling framework" section).

\section{Spatial units of analysis}

In this study, two recently developed individual specific models of activity spaces were used, namely home range model (Hasanzadeh et al. 2017), and individualized residential exposure model (Hasanzadeh et al. 2018), in addition to a more commonly used 500-m circular buffer, as the spatial units of analysis. These units were used for measurement of the built environmental factors. 
The individual home range model (IHR) is an individual-specific boundary method, which was first introduced by Hasanzadeh et al. (2017) and later implemented in a number of other studies (e.g. Kajosaari et al. 2019). Following the criteria suggested in the study by Hasanzadeh et al. (2017), all daily destinations were listed based on their distance from the participant's home location. The Jenk's optimization method revealed $4 \mathrm{~km}$ as the suitable home range distance for the data set. This distance is based on the first natural break value including at least $80 \%$ of daily destinations marked by the participants. It should be noted that the optimum number of classes for the Jenk's algorithm was determined using Goodness of Variance Fit (GVF). In the next step, a convex hull was applied to enclose all daily destinations as well as the home point. However, prior to the implementation of convex hulls, buffers were applied to each point marked by the participants. Accordingly, buffers with distances $500 \mathrm{~m}$ and $140 \mathrm{~m}$ were applied to the home locations and daily destinations respectively. According to Hasanzadeh et al. (2017), $500 \mathrm{~m}$ is the most frequently used distance for defining immediate neighborhoods in literature, and $140 \mathrm{~m}$ is identified as a suitable estimation of activity cluster sizes in a data set collected from the same area as the current study. The latter distance was calculated in the study as the average diameter of the spatial clusters formed by the aggregate of daily destinations (Hasanzadeh et al. 2017).

The second model, individualized residential exposure model (IREM), is an exposurebased model of activity spaces (Hasanzadeh et al. 2018). Following the IREM criteria (Hasanzadeh et al. 2018), we estimated the level of place exposure for each respondent throughout individual activity spaces using information on home location, daily destinations, frequency of visits, travel paths, and use of travel modes. IREM uses an inverse distance function to produce a raster representing the activity space of each individual. The raster is made of square cells with dimensions $25 \mathrm{~m} \times 25 \mathrm{~m}$, each containing a value as the estimation of exposure magnitude in the corresponding location. The boundary of IREM in this study is defined by the polygon encompassing the high exposure areas. In this study, high exposure areas were defined as places with exposure values of more than $50 \%$ of each individual's maximum exposure value.

It should be noted that all the modeling procedures for the above mentioned activity space models were implemented using the python toolbox published by Hasanzadeh (2018). In addition, two measures of elongation and centricity of activity spaces were measured to examine and control for the effect of the shape of the activity space and individuals' activity dispersion. These measures were used to test the hypothesis that the longer shaped the activity space and the more dispersed an individual's activity locations, the less the propensity to walk or cycle. Elongation is a geometric measure of activity spaces indicating the ratio of the length to width of the geometry. This captures the overall shape and, less directly, the overall extent of a person's activity space and it has been used in many studies as a proxy measure of activity dispersion (Lord et al. 2009; Perchoux et al. 2014). Centricity is a measure of the geographical distribution of activity places and can be defined as an ordinal measure of activity centers (Hasanzadeh 2019). Centricity is calculated as an index of activity space dispersion measuring the number of activity clusters formed within an individual's activity space. Consequently, individuals can be classified into three groups based on centricity of their activity spaces: monocentric: activity spaces which consist of a single cluster of activity places located in home surrounding. Bicentric: activity spaces which in addition to the cluster of activities around their home, have another center of activities somewhere further. Polycentric: activity spaces which in addition to the cluster of activities around the home, have at least two more centers of activities further from the place of residence (Hasanzadeh 2019). The third model is a circular buffer 


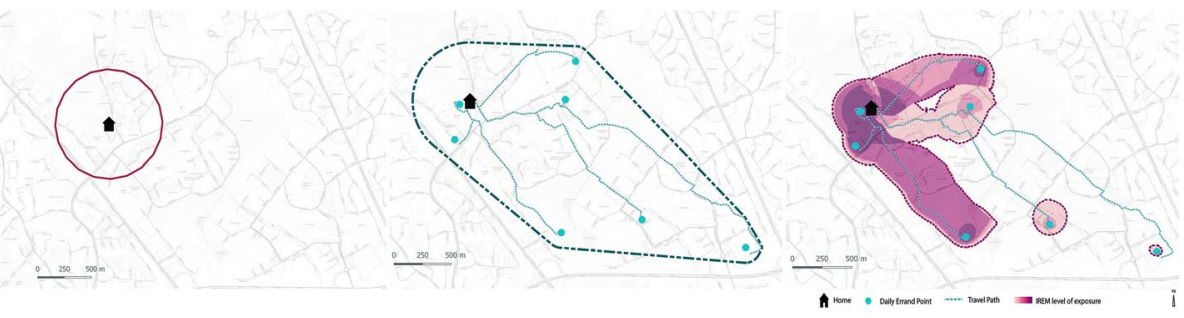

Fig. 2 The three different spatial units of analysis. From left to right: 500-m buffer, IHR, and IREM. Travel path is the shortest path between home and each destination based on network analysis

implemented in a 500-m radius around each respondent's home. Figure 2 demonstrates an example of these different spatial units of analysis for one respondent.

\section{Built environmental measures}

In order to examine to what degree and how the built environment shows to affect shopping trip mode choice of older adults when measured within different spatial units of analysis, we adapted the built environmental measures (i.e. walkability index and percentage of green area within one's neighborhood boundary) that according to the literature have shown consistent positive influence on (or correlation with) the use of active modes of transportation (for more details see "Introduction" section).

The walkability index was calculated for each individual using the three different spatial units of analysis (discussed in "Spatial units of analysis" section). It was calculated as the sum of the z-scores of the four urban form measures $[(2 \times z$-intersection density $)+(z-n e t$ residential density $)+(\mathrm{z}$-commercial floor area ratio $)+(\mathrm{z}$-land use mix $)]$. These measures are defined as follows:

- Intersection density was calculated as the share of intersections of three or more road segments per the spatial unit of analysis in question (buffer, IHR, IREM), as suggested by Frank et al. (2007). The data was drawn from the Digiroad 2017 dataset maintained by the Finnish Transport Agency. Digiroad is a national database which contains precise and accurate data on the location of all roads and streets in Finland as well as their most important physical features. The Finnish Transport Agency maintains and updates the data in the Digiroad system.

- Residential density was calculated as the ratio of the number of dwelling units to the surface area devoted to residential land use within each spatial unit of analysis in question. The residential density measure was drawn from SeutuCD 2014 building dataset (point data) which is a regional dataset provided by Helsinki Region Environmental Services Authority HSY and from the CORINE 2012 land cover dataset (raster data) that is provided for research use by Finnish environment institute. SeutuCD is an extensive collection of GIS data, which compiles the most essential register and map data sets related to the planning of the HMA. Helsinki Region Environmental Services Authority produces and provides SeutuCD data. CORINE is a $25 \times 25 \mathrm{~m}$ raster dataset that provides information on land cover. The data of CORINE 2012 has been produced as a part of the European Gioland 2012 project by Finnish Environment Institute. 
- Commercial floor area ratio was calculated as the gross commercial floor area per commercial land use. The data was drawn from SeutuCD 2014 and the Corine 2012 land cover dataset.

- Land use mix: The land use mix measure considered four land use types: residential, commercial, traffic and green space land uses. Previous studies have also considered entertainment, office, and institutional land uses (Frank et al. 2005, 2010). In this study, we adopted above mentioned land-use categories for this measure for two reasons: the availability of the data sets and because they were determined to provide the best possible correspondence to the actual built environment in the study area. The land use mix measure was calculated as follows:

$$
H=-1\left(\sum_{i=1}^{n} p i * \ln (p i)\right) / \ln (n)
$$

where $\mathrm{H}$ is the land use mix score, $p i$ is the proportion of land use $i$ among all land-use classes, and $n$ is the number of land-use types.

Although green space land use has been included in the land use mix measure, which is incorporated into the walkability index, land use mix does not provide a concrete measure of the percentage of green space within the spatial unit of analysis but rather the relative proportion of different land uses (e.g. Mavoa et al. 2018). Therefore, percentage of green space area in the three different spatial units of analysis was included in this study as an additional built environmental measure.

\section{Personal goals within the sample population}

An explanatory factor analysis (EFA) with Promax rotation and Kaiser Normalization was conducted for twelve statements measuring personal goals in SPSS 25. Four factors were identified for personal goals representing a rather high proportion $(62 \%)$ of the variance between the 12 statements. Due to low correlations with other goal variables, seven statements (out of the original 19) were left out from the final EFA after careful examination of the correlation matrix (see more details in Laatikainen et al. 2019). The four extracted factors were named based on their indicators (Table 1). Since the mode choice modelling framework used in this study (ICLV model) is able to include the ranking values of each statement directly in the model framework, we did not measure factor scores for each individual. The objective of factor analysis was to identify the underlying constructs (i.e., personal goals) and the indicators (i.e., statements) for each of these constructs to be used in the discrete choice modelling framework.

\section{Mode choice modelling framework}

Discrete choice models, which are based on the principle of utility maximization, have been often used in research to understand the factors affecting transportation mode choice. Multinomial logit (MNL) is the most commonly used model (Ramezani et al. 2018a). In such a modelling framework, the utility associated with a transportation mode includes two components - a deterministic part capturing the influence of observed potential explanatory variables such as socio-demographic, travel, attitudes, perceptions, and neighborhood 


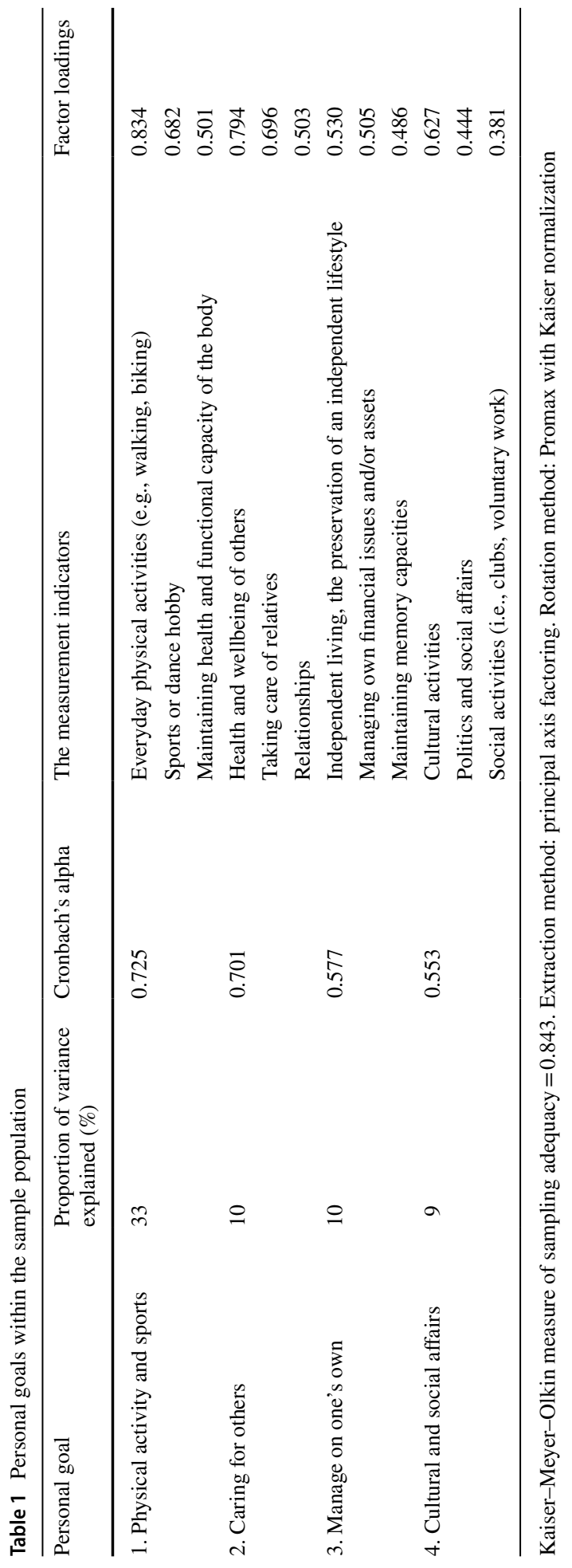


factors, and a random part capturing all other unobserved impacts (Schwanen and Mokhtarian 2005). This indicates that in an MNL model latent factors such as personal goals are treated as other observed factors. Latent factors are entered in the model as a continuous variable, which is a factor score calculated for each individual after factor analysis is conducted on ratings of a number of Likert scale statements. A number of studies have criticized this sequential process for causing spurious results and biased conclusions regarding the influence of psychological factors (e.g. Bielaire 2010). These utility maximizing models have been criticized as presenting an individual's choice process as a "black box", since the inputs are the attributes of available alternative modes of transport and the individual's characteristics, and the output is the observed choice (Ben-Akiva et al. 2002; Kamargianni et al. 2015). In order to uncover the cognitive processes inside the black box that determine choice behavior, and violate the basic axiom of utility theory, a considerable number of studies have been conducted (Kamargianni et al. 2014, 2015). During the last few decades, numerous improvements have been made in the discrete choice modelling field, which aim to better unravel the underlying process that leads to observed choice outcomes (Kamargianni et al. 2015). While using the same principle of utility maximization, these new techniques have several advantages over standard multinomial logit models. These methods are integrated in Hybrid Choice Models (HCMs). HCMs, combine " hard information" (e.g. socioeconomic characteristics) with "soft information" on population heterogeneity (e.g. psychological characteristics), and attempt to more realistically explain individual choice behavior and a substantial part of the population heterogeneity (BenAkiva et al. 2002). One of the numerous versions of HCMs is the Integrated Choice and Latent Variable (ICLV) model, which is able to model latent psychological factors such as attitudes and perceptions explicitly (Kamargianni et al. 2015) and simultaneously with the choice model. The ICLV model permits the inclusion of attitudes, opinions and perceptions as psychological latent variables in such a way that consumer behavior is better understood (Ashok et al. 2002; Ben-Akiva et al. 2002; Bolduc et al. 2005; Bhat and Dubey 2014). Such models can provide us with interesting new insights about the effect of unobservable latent factors (such as attitudes, preferences, and personal goals) and the way observable socio-demographic or even built environmental factors affect mode choice through their influence on latent factors (Ramezani et al. 2018a; Kamargianni et al. 2015). In the ICLV model, two structural equations are estimated simultaneously; one explaining the latent variables with observable characteristics (e.g. sociodemographics) using the indicators as manifestations of the latent variable, and the other one explaining the utility of each alternative with observable and unobservable latent variables (Theis 2011). Moreover, there is a measurement equation in the latent variable component of the ICLV model which is used to measure the association of indicators to a specific latent variable. The choice- and latent-variable-model components are estimated simultaneously thus achieving consistent and efficient parameter estimates (Daly et al. 2012). We follow the model structure used in Daly et al. (2012) and Potoglou et al. (2015). For details on the model structure and the maximum likelihood estimation of the ICLV model, see Daly et al. (2012) and Potoglou et al. (2015). The repeated-response (panel) nature of the data is also taken into considerations in these models (Potoglou et al. 2015).

To the authors' knowledge, the application of this modelling framework in understanding the effect of built environment on modal choice is very limited (Ramezani et al. 2018a), in spite of its abilities to give us more insight about the effects of the built environment on transportation mode choice. As Johnson et al. (2013) put it, "in the past, researchers often used main effects designs for simplicity and feasibility, and so any resulting confounding or bias were accepted as an unavoidable consequence of this choice. New methods and 
software, however, can easily construct designs that accommodate more complex model specifications',

In this study, shopping trip mode choice of the older adults was modelled using ICLV modelling structure in Python Biogeme freeware by Bierlaire (2003). Since this study examined the effect of built environment on mode choice when using three different spatial units of analysis, three different ICLV models were estimated. The factors included in all the three models were the same except for the built environmental factors, which were measured in three different spatial units of analysis (i.e., Activity spaces and buffer). Besides the built environmental factors, and the indicators of personal goals (explained in previous sections), a number of travel and sociodemographic factors were also included in the models. Table 2 presents the nature of the full set of variables included in the model estimation process. The variables that did not show significant effect were removed and final models were estimated again including only the significant variables. ${ }^{1}$ The final models were estimated with 500 numbers of draws using Modified Latin Hypercube Sampling (MLHS) method (see Hess et al. 2006). The results of the final models are presented in "Results and discussion" section. It is noteworthy that three MNL models were estimated as well to examine the statistical superiority of the ICLV models. The ICLV models were statistically superior to the MNL models and had a higher fit (i.e. percentage of the observations that the model predicts well) to the data. The Rho square bar for the MNL models were $0.213,0.211$, and 0.208 for the IREM, IHR, and $500 \mathrm{~m}$ Buffer models respectively. Moreover, in order to verify that the results do not differ significantly when using other buffer sizes than the $500 \mathrm{~m}$ buffer a robustness test was conducted. The same ICLV models were tested with built environmental variables measured in one smaller buffer $(250 \mathrm{~m})$ and one larger buffer $(750 \mathrm{~m})$ as well. Neither the fit of the models nor the parameter estimates and significance levels changed significantly when other buffer sizes were examined, compared to the $500 \mathrm{~m}$ buffer. The Rho square bar for the $250 \mathrm{~m}$ and $750 \mathrm{~m}$ models were 0.404 and 0.405 respectively. In this paper, we only present the outcomes of the final ICLV models for the IREM, IHR, and $500 \mathrm{~m}$ Buffer models (see "Results and discussion" section). However, the result of the robustness test for the buffer size is briefly presented in "Factors affecting older adults' shopping trip mode choice directly" section.

\section{Results and discussion}

The ICLV results are split into their corresponding components, including the choice (Table 3), structural equation (Table 4) and the measurement-equation (Table 5) models. These tables show the results of the three estimated ICLV models with built environmental factors calculated in the three different spatial units of analysis.

\section{Factors affecting older adults' shopping trip mode choice directly}

Table 3 presents the results of the choice model which shows factors affecting shopping trip mode choice of the sample population, directly. As illustrated in Table 3, although the

\footnotetext{
${ }^{1}$ We included the variables with $p \leq 0.05$, as significant and those with $0.05<p \leq 0.10$, as moderately significant factors in the final model. The alternative specific constants were included even if they showed larger $p$ values.
} 
Table 2 The full set of variables included in the discrete choice models

Dependent variable

Mode choice for shopping trips Nominal variable including Walk, Bike, Transit, and Car (base mode) alternatives

Built environmental factors (see "Built environmental measures" section for details)

Walkability index Continuous variable measured for three different spatial units of analysis

Green space percentage continuous variable measured for three different spatial units of analysis

Activity space measures

Elongation Continuous variable measured for IREM and IHR spatial units of analysis

Centricity Categorical variable including three categories: 1. Monocentric (base category), 2. Bicentric, 3 . Polycentric

Latent factors (see "Personal goals within the sample population" section for details)

12 Measurement indicators for 4 personal goals Ordinal variables on a scale from 0 to 6

Travel factors

Travel time of alternative $X$ (hour) Continuous variable. Travel time was estimated for each alternative mode based on the average speed of that alternative in HMA on the shortest route from home to the reported shopping place. Shortest route was found using network analyst toolbox in ArcMap. The transportation mode indicated by the participant for visiting each shopping place was taken into consideration while choosing the shortest path. The average speeds for Walking $(5 \mathrm{~km} / \mathrm{h})$, Biking $(17 \mathrm{~km} / \mathrm{h})$, and Car $(50 \mathrm{~km} / \mathrm{h})$ were obtained from Helsinki Region Transport office (HSL). The average speed for public transport $(33.4 \mathrm{~km} / \mathrm{h})$ was adopted from Salonen and Toivonen (2013). An average waiting time of 9 min based on the same reference (Salonen and Toivonen 2013) was added to the travel time by public transport

Travel cost of alternative $X$ (EURO) Continuous variable. Travel cost was estimated for Car and transit modes. For Car, it was estimated based on the shortest distance from home to the reported shopping place, and the price of gasoline at the year survey was conducted in HMA. Unfortunately, information on transit pass ownership was not available in the dataset. Cost of transit was, therefore, supposed to be the same for all respondents and equal to the cost of a one-way transit ticket.

Frequency of visit to the shopping place per month Continuous variable calculated based on reported frequency per week.

Respondent socioeconomic factors

Gender: categorical variable Dummy variable.1. Male (base), 2. Female

Age: categorical variable Dummy variable. 1. Less than 65 (base), 2. 65-75

Housing type Categorical variable. 1. Apartment, 2. Detached/terraced house (base)

Education Ordinal variable on a scale from 1 to 4 (basic education to graduate degree)

Income Ordinal variable on a scale from 1 to 5 (less than 2000 to more than 8000 euro)

Retired dummy variable 0 . No, 1 . Yes

Household structure Categorical variable. 1. Single no children (Base), 2. Single with children, 3. Couple no children, 4. Couple with children, 5. Other

Having pets Dummy variable. 0. No, 1. Yes

Having grand children Dummy variable. 0 . No, 1. Yes

Exercise regularly Dummy variable. 0. No, 1. Yes

Doing regular hobbies Dummy variable. 0. No, 1. Yes

Stated overall health Ordinal variable on a scale from 1 to 5

model in which IREM built environmental variables were included showed a very slightly better fit, it did not show statistically significant difference from the other two models. This is the same for the model with IHR measures compared to the model with the 500-m buffer measures. All the three models showed a good fit with Rho square bar of 0.405 , 0.407 , and 0.408 , for the 500-m buffer, the IHR, and the IREM, respectively. However, 


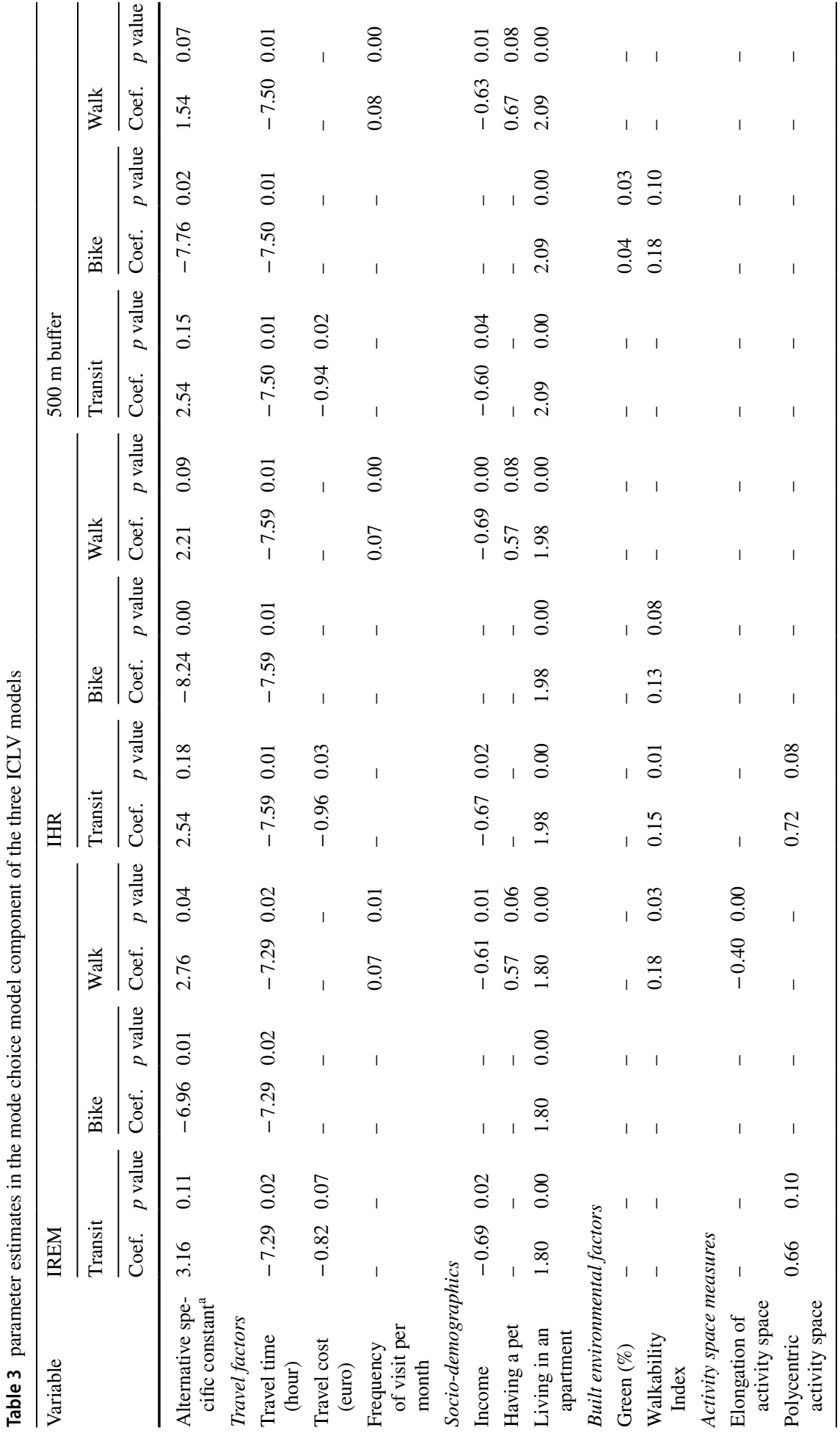




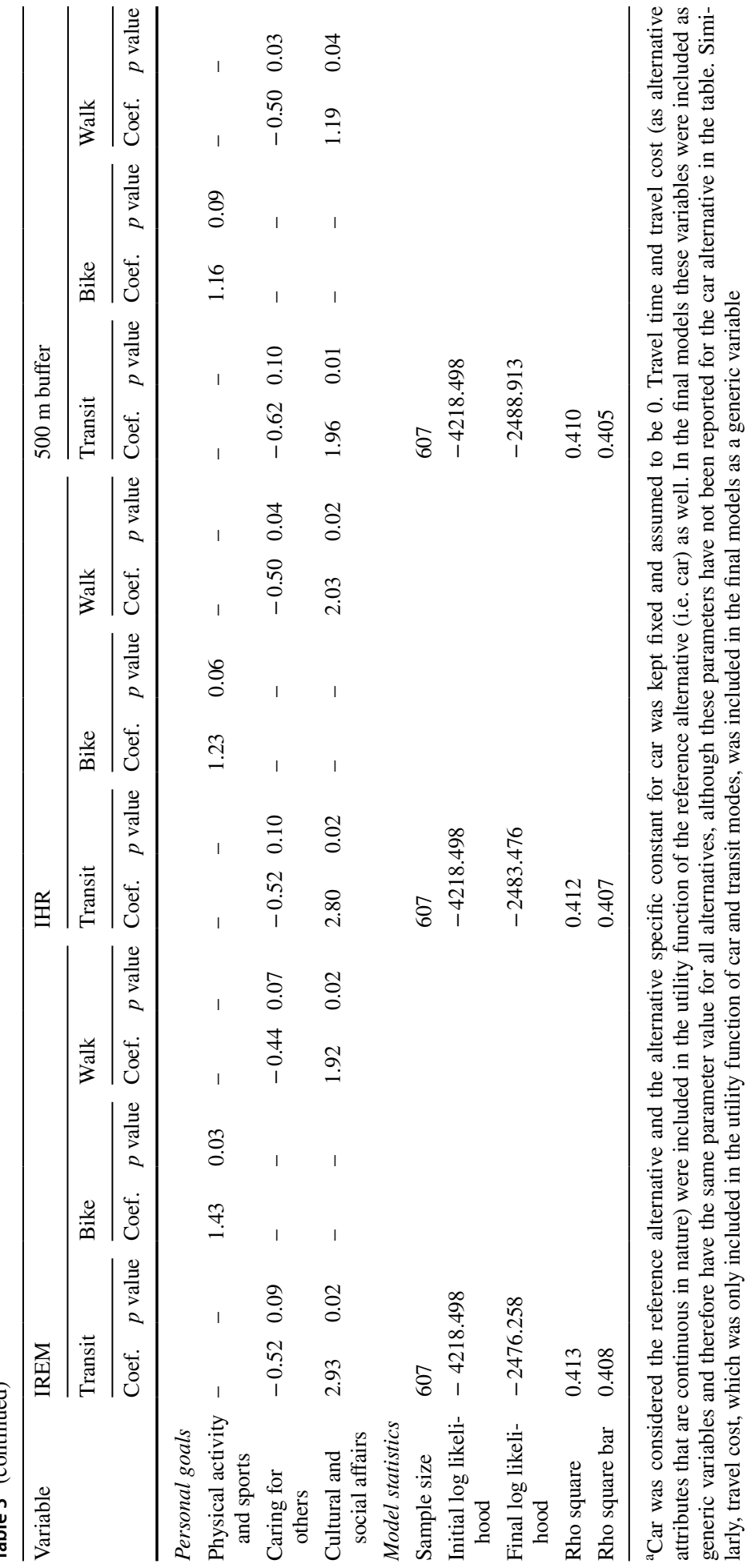




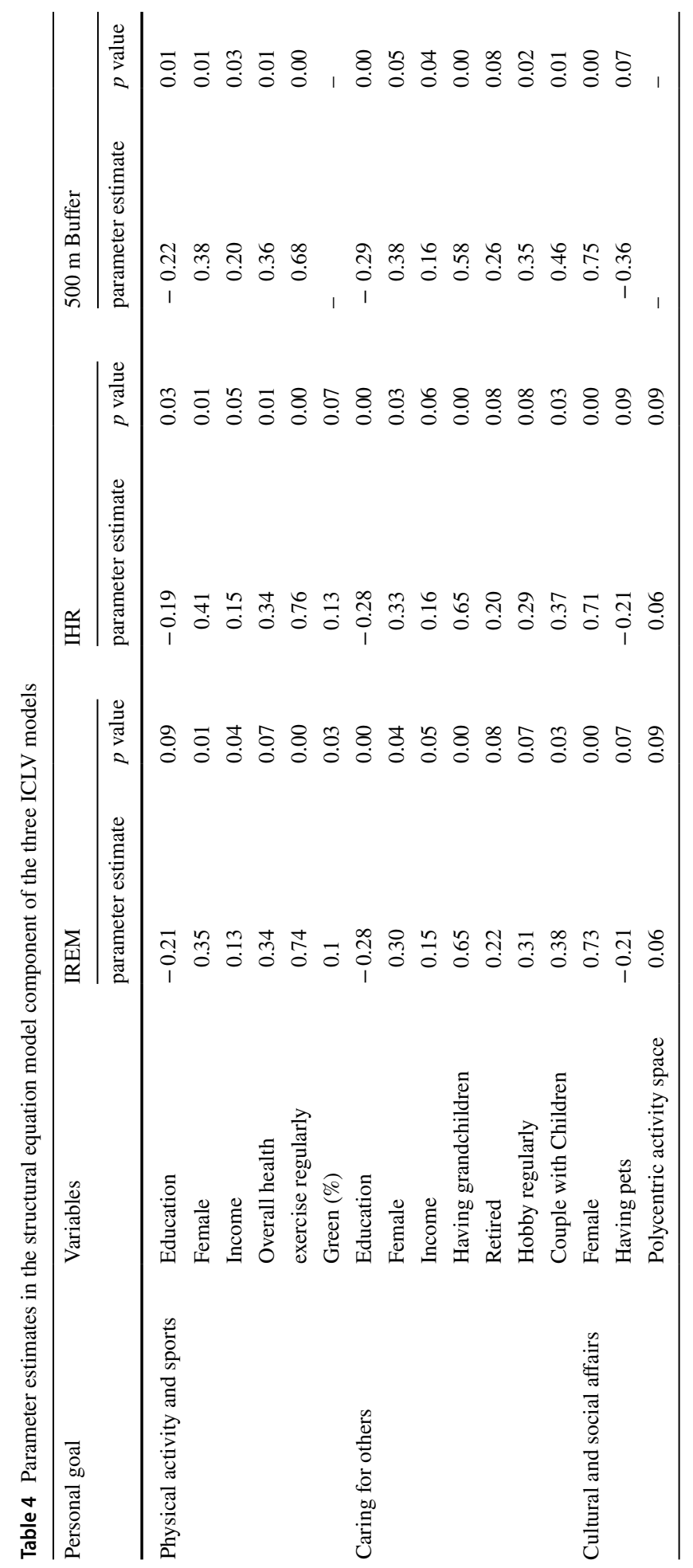




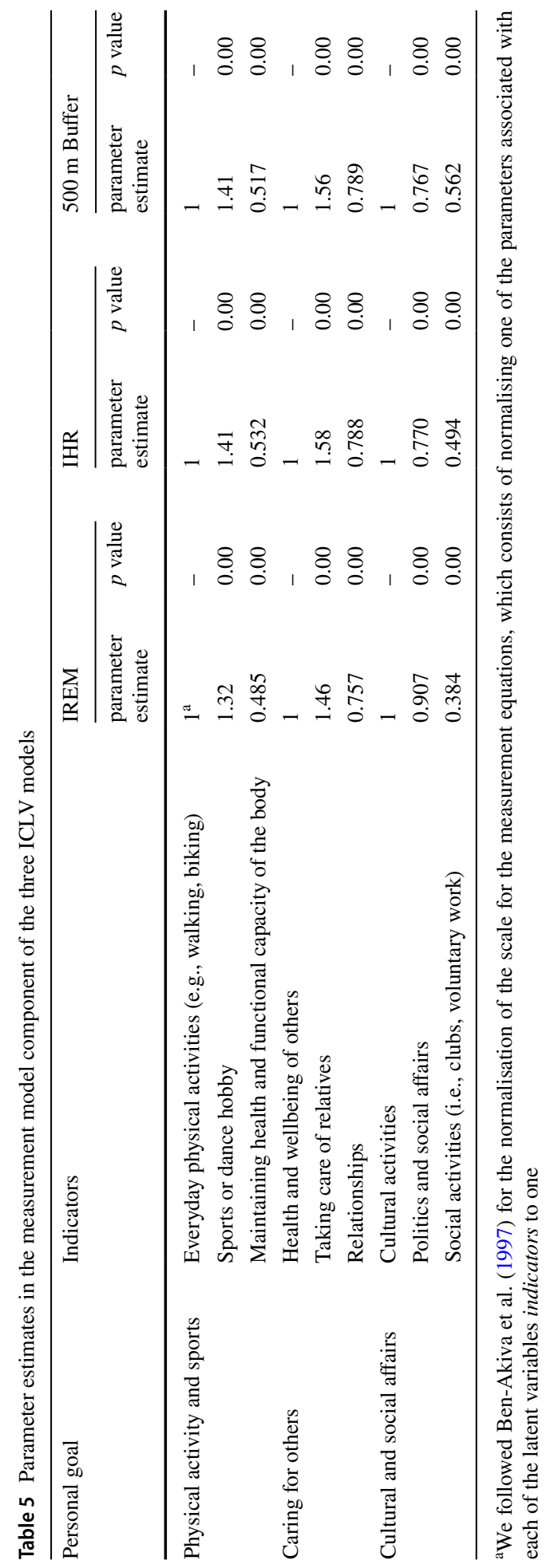


using different units of analysis for measuring built environmental factors showed to lead to different results regarding the way built environment affects shopping trip mode choice. All the other sets of variables including the travel factors, socio-demographics, and the personal goals showed consistent results in the three different models. Among the travel factors, travel time showed an equally significant negative effect on the choice of transit, walking and biking. Travel cost showed an equally significant negative effect on the choice of transit. In the process of model specification, these factors entered the models first as an alternative specific factor and then as a generic factor. A likelihood ratio test was conducted to compare the goodness of fit of the reduced models with the full models (i.e. models that included travel time and cost as an alternative specific factor). Since the models with alternative specific travel time and cost factors were not statistically superior to the models including them as generic variables, these factors were included as generic variables in the final models. This result implies that the higher the travel time or the cost (only for the transit mode) of a mode of transportation, the lower the related utility and therefore the probability for that mode to be chosen. This result is consistent with the literature on factors affecting mode choice (e.g. Ramezani et al. 2018a, b). It implies that policies to decrease the time and/or cost of travelling by sustainable modes of transportation are important if the goal is to increase the use of these modes of transportation for shopping trips of older adults. Moreover, frequency of visit per month showed a significant positive effect on walking, indicating that probability of walking to a shopping place among older adults goes higher if they visit that place more frequently. This can be due to sense of familiarity which can in turn induce perception of safety and the ability to function. However, this study did not measure such perceptions and future research is needed to confirm such conclusions regarding the effect of sense of familiarity and its association with perception of safety and the ability to function.

Among the sociodemographic factors, only income, having a pet, and living in an apartment showed significant direct effects on mode choice. Income showed a negative effect on transit use and walking but not on biking, indicating that higher income older adults have less propensity for taking transit and walking for shopping trips. However, income does not affect biking for shopping trips among older adults in HMA. Having a pet showed a significant positive effect on walking for shopping trips, which could be due to the need to walk the pets. This result is intuitive as previous research has shown that dog owners are more physically active, primarily through walking their dogs (e.g. Cutt et al. 2007), and this study shows that it can also affect their shopping trip mode choice. Living in an apartment showed a significant positive effect on transit use, walking, and biking.

Among the four personal goals, only the three goals of engaging in physical activity and sports, caring for others, and engaging in cultural and social affairs showed significant effects on shopping trip transportation mode choice of older adults. While the effect of these three goals is intuitive, the fact that the personal goal referring to the ability of older adults to manage on their own did not affect their probability of choosing more active modes of transportation is counter intuitive. Research on physical activity of this age group indicates that older adults who are confident on their abilities to manage on their own or abilities to function will be more physically active (e.g. Meisner et al. 2013), which can also affect the mode of transportation they choose for their different trip purposes, including their shopping trips. However, one explanation for this result could be that this goal can be more important for those who are worried about their functional capabilities. Thus, they may not feel confident enough to walk or cycle or take transit more than driving. The personal goal of being physically active and doing sports showed a significant positive effect on biking but not on walking or transit use. This can indicate that the older adults in HMA 
consider biking more active than walking or taking transit. This can have some implications for policymakers. For example, marketing efforts to raise awareness about the health benefits of walking and taking transit as active means of transportation can be considered as a soft policy that, for example, can change the misinterpretation that public transport is not an active means of transportation. The personal goal of caring for others showed a negative effect on walking and transit use. This might be due to the fact that driving may provide more comfort and time saving for those who feel the need to take care of others. This is also visible in the structural equation model component in which having grandchildren and being couple with children showed a positive association with this personal goal (see Table 4). Finally, the goal of engaging in Cultural and social affairs showed to have a positive effect on walking and transit use. This indicates that older adults who are more sociable are more willing to use public means of transportation or walk which provide more opportunities for socializing with people than driving. This is consistent with a growing body of research which emphasize on the relationship between social interaction and travel behavior (see Dugundji et al. 2011; Carrasco et al. 2018).

Regarding the effects of built environment, the results showed that the effects of built environmental factors (i.e. walkability index and green space percentage) on the choice of more active and sustainable modes of transportation were not consistent across the three models, in which these measures were calculated using different spatial units of analysis. Among the 500-meter buffer measures, walkability index showed a moderately significant positive effect $(p=0.10)$ and green space percentage showed a significant positive effect $(p=0.03)$ on biking. None of these measures showed an effect on walking or transit modes. Among the IHR measures, walkability index showed a moderately significant positive effect $(p=0.08)$ on biking and a significant positive effect $(p=0.01)$ on transit. Green space percentage did not show any effect on the probability of choosing any of these modes. Among the IREM measures, however, the walkability index showed a significant positive effect $(p=0.03)$ on walking but not on any of the other modes. Same as what was seen in the model with IHR measure, exposure to green spaces did not show any direct significant effect on shopping trip mode choice in the model with IREM measures.

The significance level of the effect of walkability index on mode choice was higher in the two activity space models compared to the 500-meter buffer. This result is consistent with a growing body of literature which point to the influence of the spatial unit of analysis used on the results obtained regarding the influence of built environment on travel behavior (e.g. Mitra and Buliung 2012; Clark and Scott 2014). The results of this study show that the individual-based activity spaces can provide us with a better understanding of the effect of built environment on travel behavior. As Laatikainen et al. (2018) conclude, these methods provide us with insights into the way the actual exposure to the built environmental characteristics can affect travel behavior. In the study by Mitra and Buliung (2012) who investigated the influence of the scale of the spatial unit of analysis on the results we obtain regarding the effect of environmental factors on mode choice, less environmental factors showed significant effect on active transportation mode choice when buffer sizes larger than $400 \mathrm{~m}$ buffer (e.g. 800-1000-m buffers) were used as spatial units of analysis. However, our study found that the environmental measures show more significant effect on sustainable mode choice (either directly or through their influence on the personal goal of being physically active) when measured in individuals' activity spaces, although these activity spaces have a larger scale than a $500 \mathrm{~m}$ buffer (the $p$ value for the effect of walkability index on mode choice for the $500 \mathrm{~m}$ buffer was 0.1 while the $p$ value of the effect of walkability index for IREM and IHR models were between 0.01 and 0.03 ). 
Moreover, the walkability index measure showed to have a very significant positive effect on walking for shopping trips in the IREM model while in IHR and buffer models it showed significant effect on transit use and/or biking. This result is interesting as IREM boundary is shaped based on the concept of maximum exposure of an individual to the built environment around his activity places (including one's residential environment, the environment around all the common activity places, and the routes between home and activity places). This maximum exposure is measured based on the mode of transportation used and the frequency of visit, giving highest weights to the routes and areas people walk in and visit more frequently and the lowest weights to the ones they drive in and visit less frequently. Therefore, the result of this study shows that although the discrete choice model fit does not improve significantly, when walkability index is measured in this more dynamic person based spatial unit of analysis, the information that the model provides is more intuitive (i.e. higher exposure to walkable built environment leads to higher probability of walking). This can also explain the diverse results in the literature regarding the influence of built environmental factors on active travel behavior (see Ewing and Cervero 2010), pointing to the influence of the spatial unit of analysis and the way exposure to built environmental characteristics are usually measured. The study, therefore, supports the literature cautioning against the use of residential buffers to assign built environment characteristics (Perchoux et al. 2013; Holliday et al. 2017).

As discussed in "Mode choice modelling framework" section, a robustness test was run to verify that the results do not differ significantly when using other buffer sizes. Similar to the results obtained from the $500 \mathrm{~m}$ buffer model, walkability index and the green space percentage showed significant effect on biking in these two additional models. The fit of the model and the significance level for the influence of walkability index and green space percentage remained the same for the $750 \mathrm{~m}$ buffer. The walkability index showed a slightly more significant effect on biking when measured in $250 \mathrm{~m}$ buffer compared to when measured in $500 \mathrm{~m}$ buffer (i.e. $p$ value of 0.09 for this parameter in the $250 \mathrm{~m}$ buffer model compared to 0.10 in the $500 \mathrm{~m}$ buffer model). However, the fit of the $250 \mathrm{~m}$ model to the data was slightly lower (Rho square bar of 0.404 compared to 0.405 in the $500 \mathrm{~m}$ buffer model) and the results regarding the influence of walkability index on biking rather than walking trips remained the same. This robustness test confirms that the result we obtained regarding the priority of activity space models to the commonly used buffers as spatial units of analysis does not differ when other buffer sizes than the $500 \mathrm{~m}$ buffer are used.

In the models including activity space measures, two other measures of shape and dispersion of activity spaces were included as well-namely elongation and centricity. Elongation, which is a measure of the shape of an individual's activity space, did not show any significant effects in the model with IHR measures. However, it did show a significant negative effect on walking in the model with IREM measures, meaning that as the ratio of length to width of an older adult's activity space grows the probability of choosing to walk for shopping trips diminishes.

Polycentricity, which is a measure of the dispersion of activity spaces (see "Spatial units of analysis" section), showed a significant positive effect on transit use for both models with IHR and IREM measures. This result is interesting showing that individuals who have their frequent activities distributed between several locations (they have several activity clusters) tend to favor the use of public transport. However, this result does not provide any information about the effect of built environmental characteristics of those polycentric activity spaces, although a previous study by Hasanzadeh (2019) found that the activity space of those living in suburban areas of HMA tends to be polycentric. 
Moreover, although elongation and polycentricity, and built environmental factors of individuals' activity spaces showed significant effects on shopping trip mode choice, the causality directions might be more complex. For example, elongation of activity space might be a proxy for preference for, and more regular use of, certain modes of transportation, with those having a preference for active modes of transportation choosing activity locations closer to home, which can reduce the size and shape (or the ratio of length to width) of their activity space compared to those with preference for, and more regular use of, motorized modes of transportation. Similarly, although those with polycentric activity space may have higher probability to choose public transport for their shopping trips, it is also possible that those who have a preference for public transport or use public transport more regularly than car, may cluster their activities around several locations (i.e. home, work, or station nodes). Moreover, although urban form characteristics of the activity space (e.g. walkability index) showed a significant effect on walking for shopping transport, this is also probable that people who have a preference for walking self-select areas for their residential location as well as other activity locations that make walking for shopping trips possible. While inclusion of personal goals of older adults in this study could to some extent account for the influence of preferences for specific modes of transportation, and therefore possible self-selection biases, it is also possible that the same latent psychosocial factors can affect several outcome variables (i.e. residential location choice, activity location choice, and mode choice). The results of this study point to the need to account and control for more individual-based travel characteristics when trying to understand the relationships between built environment and travel behavior and show that the dynamic person based activity spaces (e.g. IREM) are able to reveal such information about personenvironment interactions that could not be otherwise explained. However, future studies should use research designs and analysis methods that could better account for and unravel the above mentioned self-selection biases and the relationships between several dependent outcomes, rather than just focus on transportation mode choice as an outcome of activity space attributes and personal goals.

\section{Factors affecting older adults' personal goals}

Table 4 presents the Parameter estimates in the structural equation model component of the ICLV model. The results of the ICLV model provided an understanding of the effects of observed factors such as built environmental factors and socio-demographics on personal goals, which in turn affect transportation mode choice of the older adults.

It was found that although some of the observed factors do not show a direct significant effect on mode choice, they do affect the personal goals that directly influence mode choice. For example, while percentage of green space exposure did not show any direct influence on mode choice when measured using IREM and IHR activity space models, it did show to have a positive effect on the goal of being physically active which increases the probability of biking for shopping trips. Similarly, many of the sociodemographic factors that did not show to affect the use of more active modes of transportation for shopping trips directly, had an influence on older adults' personal goals that affect mode choice. For example, being a female showed to have a positive effect on the personal goals of engaging in social and cultural activities (which positively influences walking and transit use) and being more physically active (which positively influences biking). On the other hand, it negatively affected the goal of caring for others which in turn has a negative influence on walking and transit use. These additional parameters identified by the ICLV model, which 
provide valuable information that cannot be captured from the reduced form multinomial logit models (Vij and Walker 2012), can be employed for policy analysis. While some of these personal goals are not easy to change, some lessons can be learnt for policymaking. For example, as discussed above, the results of this study found that being a female has a positive effect on having the goal of being physically active, which eventually affects sustainable mode choice. Policy makers can use this information in different ways. This can include providing feedback to female residents regarding the value of being physically active to increase the effect of this personal goal on the propensity to use more sustainable modes of transportation among this segment of the population who have more probability to set this as a personal goal. Another strategy would be to target male residents and disseminate knowledge about the personal benefits of being physically active to reduce the gap in propensity for being physically active among this group of residents.

Moreover, the information provided regarding the influence of green space exposure when measured in IREM and IHR, and not in 500-m buffer, on having the personal goal of being physically active can indicate that the immediate surrounding of one's home, one's common destinations, and the paths taken to reach those destinations (i.e. the maximum exposure area) is more important in setting up personal goals. We can also conclude that providing more green space in cities can lead to the use of more active modes of transportation for older adults, even if it does not show a direct effect. However, similar to the discussions on self-selection issues in "Factors affecting older adults' shopping trip mode choice directly" section, the issue of uncertainty about causality direction holds for the effect of green space percentage on the goal of being physically active as well. While this study provides insights about associations between green space exposure and the personal goal of being physically active, it is also possible that those who have a personal goal of being physically active in mind, self-select not only a green residential environment (residential self-selection), but also green leisure destinations and/or green routes into their activity locations. Further study is needed to examine the possibility of such causality directions.

It is also worth mentioning that as Haustein (2012) emphasizes, soft policy measures (e.g. the effect of campaigns to spread information about benefits of physical activity) are often overlooked compared to the hard measures such as barrier-free accessibility, and other urban form or level of service measures, especially when looking at the older adults. Today, the older adults have different lifestyles and only a small percentage can be described as mobility-impaired (Haustein 2012). The more active subgroups of seniors have requirements, and needs that go beyond barrier free access and there is a probability that change in their travel behavior (i.e. a shift from car use to the choice of more sustainable and active means of transportation) can be achieved by targeting their psychological characteristics (e.g. their personal goals). The results of this study provide some insights in this regard and emphasize the need for further research to explore the effects of such factors on travel behavior of this age group.

Table 5 presents the association of each of the indicators with the corresponding latent variable (personal goal) as the result of the measurement equation in the ICLV model. The results indicate that the latent personal goal of doing physical activity and sport is positively associated with all the three indicators with the highest rates attributed to the sports and dance hobby indicator when compared with the other two indicators. This result regarding the positive association of indicators with personal goal is the same for all the other two latent variables as well. For the personal goal of caring for others taking care of relatives shows to have received the highest ratings. For the personal goal of cultural and social affairs, the cultural activities indicator receives the highest rates. Such information gained through the use of ICLV model structures can also help in selecting the most 
relevant indicators for measurement of latent factors when including all indicators in a survey is not possible (e.g. due to the length of survey).

\section{Conclusions}

This paper analyzed shopping trip mode choice of older adults in Helsinki Metropolitan Area. It sought to provide a better understanding of the way built environment can affect travel behavior by using two activity space models, in addition to the commonly used buffers around home location, as spatial units of analysis. Moreover, it used an ICLV modelling framework to unravel more complex relationships between observable and latent factors that can affect travel behavior of this age group and to investigate whether personal goals of older adults affect their shopping trip mode choice.

A number of sociodemographic, built environmental, psycho-social, and travel factors showed to affect shopping trip mode choice of older adults. While the aim of this study was not to come up with concrete policies, the information gained through this study can provide some insights for policy making. For example, the study showed that even after controlling for the effect of personal goals, higher exposure to dense, mixed use and green areas increase the propensity to use more sustainable modes of transportation for shopping trips among older adults in HMA. Little attention has been paid to the implications of such land use policies for older adults (Cao et al. 2010). The results of this study indicate that although the needs of the older adults are different from those of other segments of the population, policies to increase density, land use diversity and green land uses can increase the use of more active and sustainable modes of transportation among this age group, similar to what has been commonly discussed in the literature on the effect of built environment on travel behavior of other age groups. Moreover, the use of ICLV modelling framework in this study, helped in attaining information that could not be otherwise gained. This study provided information about the segments of the older population that would be more sensitive to soft policies, such as information campaigns targeting latent personal goals of the older adults. For example, this study suggests that female older adults in HMA might have more sensitivity to information campaigns that would highlight the health benefits of the use of transit as an active mode of transportation, as discussed in the previous section. As Haustein (2012) puts it, a necessary condition to successfully and efficiently implement information campaigns is that measures are developed that address specific target groups rather than all potential user groups at once. This study, therefore, provides such insights needed for policy makers that could not be gained through the use of simple multinomial logits or regression models.

In addition to the insights provided for policy making, this study contributed to the literature on the effects of built environment on travel behavior by providing information on how the use of three different spatial units of analysis (i.e. two different activity space models versus a commonly used buffer) can affect the results we obtain regarding the influence of built environment on transportation mode choice. One of the key results of this study was that although the use of more person based dynamic spatial units of analysis did not significantly increase the fit of the ICLV models, it did show an increase in the significance level of the effect of walkability index on travel behavior, as compared to a buffer. In other words, it provided different, and more intuitive, results regarding the way built environment affects travel behavior (e.g. the positive and more significant effect of walkability index on walking trips in IREM model compared 
to buffer model where there was a moderately significant effect on biking). Moreover, when measuring built environment using the dynamic activity space models, the built environmental measure of green space exposure showed a positive influence on the older adults' personal goal of doing physical activity and sports, which in turn affected travel behavior. This was not shown when using the static and commonly used buffer as the spatial unit of analysis. Furthermore, the study found that individuals' activity space characteristics, such as the shape and dispersion of activity space, may affect the mode of transportation that they choose for their shopping trips. These results show possible priority of using dynamic activity space models to static buffers.

However, this study is not void of limitations and a number of future research directions can be highlighted. First, whether the effect of exposure to the built environmental factors on travel behavior is more pronounced for older people than for other age groups is unknown, as we have focused on this age group in this study. Since this study is the first to use IREM and IHR measures in mode choice models, the results cannot be generalized. Future studies in other contexts, and involving other age groups should be conducted before any generalization can be made regarding the priority of these dynamic activity spaces in understanding the way built environment affects mode choice. Second, while having several advantages over other activity space models in the literature (see Hasanzadeh et al. 2018), one limitation of the activity space models used in this study is that they rely on the shortest path and do not include the actual route taken from home to activity places. Moreover, trip chaining is not accounted for. While this makes the models less data-hungry and therefore applicable to common data collected in research and practice (for example, travel diaries collected in national travel surveys), it does not provide accurate information specially when trying to understand the effect of the degree of exposure to the built environmental factors on travel behavior. Future research should collect more accurate data to investigate the effect of exposure to the routes between different activity places. Such data could be collected, for example, by GPS devices or travel tracking apps. Another possibility is to use map-based survey tools such as the one used in this study, through which respondents can mark the routes they usually take to reach their destinations. Having obtained such detailed travel behavior data, IREM can be modified to represent actual exposure to the routes between different activity places. Furthermore, although the data set used in this study includes older adults that are not yet retired (i.e. 55-65-year-olds), the respondents have not been directly asked to report their work place location. Although the respondents might have reported their work place location under the category of "offices, bureaus, and businesses", future studies should ask about work place location directly to increase data accuracy. Third, a number of studies in the literature (e.g. Mitra and Buliung 2012; Clark and Scott 2014) have discussed the possible effect of the scale of spatial units of analysis (i.e. Modifiable Areal Unit Problem referred to as MAUP) on the results obtained regarding the effect of built environment on travel behavior. For example, measurements such as land use mix, which is included in the walkability index, can be particularly prone to MAUP. Land use mix score can widely differ based on the shape and scale of the activity space of the individual as we are aggregating the land use data layer that has a resolution of $25 \mathrm{~T} \times 25 \mathrm{~m}$ (raster dataset) to larger and dynamic spatial units (i.e. dynamic activity space which has different shapes and scales for different individuals). This study, to some extent, accounted, for the effect of the differing shapes of the dynamic activity spaces by including a control variable (i.e. elongation). Future studies could come up with more robust methods to resolve the MAUP that may still exist due to the different resolutions of the land use data layer (the raster dataset) and the different spatial units of analysis. 
Fourth, although this study showed a positive effect of polycentricity of activity spaces on the use of public transport, it did not investigate possible interactions between centricity and walkability index (i.e. the effect of built environmental characteristics of these polycentric activity spaces). Future research could include such interactions to investigate whether those exposed to walkable environment (or other built environmental measures such as greenery) in one or more centers of activities further from their place of residence would have higher propensity for walking, cycling or transit use. Fifth, this study used the commonly used walkability index which considers only the degree of urbanization. However, the spatial needs of the older adults may be more demanding (Metz 2003). Future studies should include more detailed physical environment measures such as safe crossing points, resting places, and equal pavement surfaces to see to what degree and how those characteristics of activity spaces could affect mode choice decisions of older adults. In addition, it should be noted that the land use mix measure used in this study did not include all possible land uses such as public spaces, water bodies and agricultural land, which might have affected the land use mix score. Future studies should include these additional land uses as well. Furthermore, other factors such as type of shopping trip and carrying loads can affect shopping trip and future studies should consider inclusion of such factors in mode choice models. Last but not least, as discussed in the previous section, the issue of causality versus self-selection of residential location as well as activity locations should be studied in future using research designs and analysis methods that allow for examination of different causality directions between several independent and outcome variables. Although ICLV models have some priorities over the simple multinomial models, and can to some extent account for self-selection bias by including individuals' attitudes and personal goals, one limitation of these models developed for discrete choice analysis, is that in ICLV models the standard estimation methodology is the method of maximum likelihood estimation. When there are many binary/ordered-response outcomes (e.g. indicators of personal goals) and/or a nominal variable, the integrals in the overall probability expression are computed using simulation techniques. As indicated by Hoshino and Bentler (2013), this can 'be difficult to impossible when the model is complex or the number of variables is large." (Bhat 2015). To address these issues, Bhat and Dubey (2014) and Bhat (2015) have developed a new econometric approach that they label as the generalized heterogeneous data model (GHDM), which is according to them general enough to accommodate other models in the literature as special cases. One possibility is to use this approach in future, for example, for the estimation of joint mixed models that could include multiple discrete choice outcomes (residential location choice, destination location choice and mode choice). Using the GHDM approach in future studies, these outcomes can be modeled together by specifying latent underlying unobserved individual psycho-social factors that impact the many outcomes, and generate the jointness among the outcomes.

Acknowledgement Open access funding provided by Aalto University.

Authors' contribution SR: Literature search and review, research question conception, content planning, data preparation, discrete choice modelling, main manuscript writing, reviewing, and editing. TL: Data preparation, factor analysis, GIS analysis, partial manuscript writing. KH: Data preparation, activity space modelling, GIS analysis, partial manuscript writing. MK: Manuscript reviewing, general guidelines.

\section{Compliance with ethical standards}

Conflict of interest On behalf of all authors, the corresponding author states that there is no conflict of interest. 
Open Access This article is distributed under the terms of the Creative Commons Attribution 4.0 International License (http://creativecommons.org/licenses/by/4.0/), which permits unrestricted use, distribution, and reproduction in any medium, provided you give appropriate credit to the original author(s) and the source, provide a link to the Creative Commons license, and indicate if changes were made.

\section{References}

Alsnih, R., Hensher, D.: The mobility and accessibility expectations of seniors in an aging population. Transp. Res. A 37(10), 903-916 (2003)

Ashok, K., Dillon, W.R., Yuan, S.: Extending discrete choice models to incorporate attitudinal and other latent variables. J. Market. Res. 39(1), 31-46 (2002)

Banerjee, T., Baer, W.C.: Beyond the Neighborhood Unit: Residential Environment and Public Policy. Plenum Press, New York (1984)

Banister, D., Bowling, A.: Quality of life for the elderly: the transport dimension. Transp. Policy 11, 105-115 (2004)

Barber, G.: Aggregate characteristics of urban travel. In: Hanson, S. (ed.) The Geography of Urban Transportation, pp. 81-99. The Guilford Press, New York (1995)

Ben-Akiva, M., Walker, J., Bernardino, A.T., Gopinath, D.A., Morikawa, T., Polydoropoulou, A.: Integration of Choice and Latent Variable Models. Massachusetts Institute of Technology, Cambridge (1997)

Ben-Akiva, M., Walker, J., Bernardino, A.T., Gopinath, D.A., Morikawa, T., Polydoropoulou, A.: Integration of choice and latent variable models. In: Mahmassani, H.S. (ed.) Perpetual Motion: Travel Behaviour Research Opportunities and Application Challenges, pp. 431-470. Elsevier, Amsterdam (2002)

Bhat, C.R.: A new generalized heterogeneous data model (GHDM) to jointly model mixed types of dependent variables. Transp. Res. Part B 79, 50-77 (2015)

Bhat, C.R., Dubey, S.K.: A new estimation approach to integrate latent psychological constructs in choice modeling. Transp. Res. Part B 67, 68-85 (2014)

Bielaire, M.: Estimating hybrid choice models with the new version of Biogeme, Seminar series. No. EPFLTALK-152415 (2010)

Bierlaire, M.: Biogeme: a free package for the estimation of discrete choice models. In: Proceedings of the 3rd Swiss Transportation Research Conference, Ascona (2003)

Böcker, L., van Amen, P., Helbich, M.: Elderly travel frequencies and transport mode choices in Greater Rotterdam, the Netherlands. Transportation 44, 831-852 (2017)

Bolduc, D., Ben-Akiva, M., Walker, J., Michaud, A.: Hybrid choice models with logit kernel: applicability to large scale models. In: Lee-Gosselin, M., Doherty, S. (eds.) Integrated Land-Use and Transportation Models: Behavioral Foundations, pp. 275-302. Elsevier, Oxford (2005)

Boschmann, E., Brady, S.: Travel behaviors, sustainable mobility, and transit-oriented developments: a travel counts analysis of older adults in the Denver. Colorado metropolitan area. J. Transp. Geogr. 33(C), 1-11 (2013)

Cao, X., Mokhtarian, P.L., Handy, S.L.: Neighborhood design and aging: an empirical analysis in northern California. Int. J. Sustain. Transp. 4(6), 347-371 (2010)

Carrasco, J.A., Axhausen, K., Dugundji, E.: Social interactions and travel behavior, (Editorial). Res. Transp. Econ. 68, 1 (2018)

Chen, I.W.H., Gross, F., Pecheux, K., Jovanis, P.P.: Estimating modal preference between ITS-enhanced and existing paratransit services. In: Paper Presented at the 2004 Annual Meeting of the Transportation Research Board, Washington, DC (2004)

Clark, A., Scott, D.: Understanding the impact of the modifiable areal unit problem on the relationship between active travel and the built environment. Urban Stud. 51(2), 284-299 (2014)

Coughlin, J.F., D'Ambrosio, L.A.: Aging America and Transportation: Personal Choices and Public Policy, p. 288. Springer, New York (2012)

Cutt, H., Giles-Corti, B., Knuiman, M., Burke, V.: Dog ownership, health and physical activity: a critical review of the literature. Health Place 13(1), 261-272 (2007)

Daly, A., Hess, S., Patruni, B., Potoglou, D., Rohr, C.: Using ordered attitudinal indicators in a latent variable choice model: a study of the impact of security on rail travel behavior. Transportation $39(2)$, 267-297 (2012)

Ding, C., Cao, X., Wang, Y.: Synergistic effects of the built environment and commuting programs on commute mode choice. Transp. Res. Part A Policy Pract. 118, 104-118 (2018) 
Dugundji, E.R., Páez, A., Arentze, T.A., Walker, J.L., et al.: Transportation and social interactions. Transp. Res. Part A Policy Pract. 45(4), 239-247 (2011)

European Commission: The 2015 Ageing Report: underlying assumptions and projection methodologies. Joint Report prepared by the European Commission (DG ECFIN) and the Economic Policy Committee (AWG) (2014). https://doi.org/10.2765/76255

European Commission: Eurostat regional yearbook 2018 (2018). https://doi.org/10.2785/23197

Ewing, R., Cervero, R.: Travel and the built environment: a meta-analysis. J. Am. Plan. Assoc. 76(3), 265294 (2010)

Farber, S., O’Kelly, M., Miller, H.J., Neutens, T.: Measuring segregation using patterns of daily travel behavior: a social interaction based model of exposure. J. Transp. Geogr. 49, 26-38 (2015)

Frank, L.D., Schmid, T.L., Sallis, J.F., Chapman, J., Saelens, B.E.: Linking objectively measured physical activity with objectively measured urban form: findings from SMARTRAQ. Am. J. Prev. Med. 28(2), 117-125 (2005)

Frank, L., Kerr, J., Chapman, J., Sallis, J.: Urban form relationships with walk trip frequency and distance among youth. Am. J. Health Promot. 21(4_suppl), 305-311 (2007)

Frank, L.D., Sallis, J.F., Saelens, B.E., Leary, L., Cain, L., Conway, T.L., Hess, P.M.: The development of a walkability index: application to the neighborhood quality of life study. Br. J. Sports Med. 44(13), 924-933 (2010)

Freund, A., Riediger, M.: Goals as building blocks of personality and development in adulthood. In: Mroczek, T., Little, D.K. (eds.) Handbook of Personality Development, pp. 353-372. Laurence Erlbaum Associates, New York (2006)

Gould, J., Golob, T., Barwise, P.: Why do people drive to shop? Future travel and telecommunications tradeoffs. Paper UCI-ITS-AS-WP-98-1, posted at the eScholarship Repository, Center for Activity Systems Analysis, University of California (1998). http://repositories.cdlib.org/cgi/viewconten t.cgi? article $=1035 \&$ context $=$ itsirvine $/$ casa

Hahn, J.S., Kim, H.C., Kim, J.K., Ulfarsson, G.F.: Trip making of older adults in Seoul: differences in effects of personal and household characteristics by age group and trip purpose. J. Transp. Geogr. 57, 55-62 (2016)

Hasanzadeh, K.: IASM: Individualized activity space modeler. SoftwareX 7, 138-142 (2018)

Hasanzadeh, K.: Exploring centricity of activity spaces: from measurement to the identification of personal and environmental factors. Travel Behav. Soc. 14, 57-65 (2019)

Hasanzadeh, K., Broberg, A., Kyttä, M.: Where is my neighborhood? A dynamic individual-based definition of home ranges and implementation of multiple evaluation criteria. Appl. Geogr. 84, 1-10 (2017)

Hasanzadeh, K., Laatikainen, T., Kyttä, M.: A place-based model of local activity spaces: individual place exposure and characteristics. J. Geogr. Syst. 20(3), 227-252 (2018)

Haustein, S.: Mobility behavior of the elderly: an attitude-based segmentation approach for a heterogeneous target group. Transportation 39(6), 1079-1103 (2012)

Hess, S., Train, K.E., Polak, J.W.: On the use of a modified latin hypercube sampling (MLHS) method in the estimation of a mixed logit model for vehicle choice. Transp. Res. B 40, 147-163 (2006)

Hjorthol, R.J., Levin, L., Sirén, A.: Mobility in different generations of older persons: the development of daily travel in different cohorts in Denmark, Norway and Sweden. J. Transp. Geogr. 18, 624-633 (2010)

Holliday, K.M., Howard, A.G., Emch, M., Rodriguez, D.A., Evenson, K.R.: Are buffers around home representative of physical activity spaces among adults? Health Place 45, 181-188 (2017)

Horton, F.E., Reynolds, D.R.: Effects of urban spatial structure on individual behavior. Econ. Geogr. 47(1), 36-48 (1971)

Hoshino, T., Bentler, P.M.: Bias in factor score regression and a simple solution. In: De Leon, A.R., Chough, K.C. (eds.) Analysis of Mixed Data: Methods \& Applications, pp. 43-61. CRC Press, Boca Raton (2013)

Jaakola, V., Saarto, H., Sundström A.: Helsinki facts and figures 2018. Helsinki (2018). https://www.hel. fi/hel2/tietokeskus/julkaisut/pdf/18_12_11_tasku18_en_net.pdf

Johnson, R., Lancsar, E., Marchal, D., et al.: Constructing experimental designs for discrete-choice experiments: report of the ISPOR conjoint analysis experimental design good research practices task force. Value Health 16, 3-13 (2013)

Kajosaari, A., Hasanzadeh, K., Kyttä, M.: Residential dissonance and walking for transport. J. Transp. Geogr. 74, 134-144 (2019)

Kamargianni, M., Ben-Akiva, M., Polydoropoulou, A.: Incorporating social interaction effects into hybrid choice models. Transportation 41(6), 1263-1285 (2014) 
Kamargianni, M., Dubey, S., Polydoropoulou, A., Bhat, C.: Investigating the subjective and objective factors influencing teenagers' school travel mode choice-an integrated choice and latent variable model. Transp. Res. Part A 78, 473-488 (2015)

Kemperman, A.D.A.M., Timmermans, H.J.P.: Green spaces in the direct living environment and social contacts of the aging population. Landsc. Urban Plan. 129, 44-54 (2014)

Kim, S.: Assessing mobility in an aging society: personal and built environment factors associated with older people's subjective transportation deficiency in the US. Transp. Res. Part F 14, 422-429 (2011)

Kim, S., Ulfarsson, G.F.: Travel mode choice of the elderly: effects of personal, household, neighborhood, and trip characteristics. Transp. Res. Rec. 1894(1), 117-126 (2004)

Krizek, K.L.: Residential relocation and changes in urban travel: Does neighborhood-scale urban form matter? J. Am. Plan. Assoc. 69(3), 265-281 (2003)

Laatikainen, T.E., Hasanzadeh, K., Kyttä, M.: Capturing exposure in environmental health research: challenges and opportunities of different activity space models. Int. J. Health Geogr. 17(1), 29 (2018)

Laatikainen, T.E., Haybatollahi, M., Kyttä, M.: Environmental, individual, and personal goal influences on older adults' walking in the Helsinki metropolitan area. Int. J. Environ. Health 16, 58 (2019)

Liu, W., Lu, H., Sun, Z., Liu, J.: Elderly's travel patterns and trends: the empirical analysis of Beijing. Sustainability 9, 981 (2017)

Lord, S., Joerin, F., Thériault, M.: La mobilité quotidienne de banlieusards vieillissants et âgés: Déplacements, aspirations et significations de la mobilité. Le Géogr. Can. 53(3), 357-375 (2009)

Lu, Y.: Using google street view to investigate the association between street greenery and physical activity. Landsc. Urban Plan. (in press) (2018). https://doi.org/10.1016/j.landurbplan.2018.08.029

Mavoa, S., Boulangé, C., Eagleson, S., Stewart, J., et al.: Identifying appropriate land-use mix measures for use in a national walkability index. J. Transp. Land Use 11(1), 681-700 (2018)

Meisner, B.A., Weir, P.L., Baker, J.: The relationship between aging expectations and various modes of physical activity among aging adults. Psychol. Sport Exerc. 14, 569-576 (2013)

Mercado, R., Páez, A., Scott, D.M., Newbold, K.B., Kanaroglou, P.: Transport policy in aging societies: an international comparison and implications for Canada. Open Transp. J. 1, 1-13 (2007)

Metz, D.: Transport policy for an ageing population. Transp. Rev. 23(4), 375-386 (2003)

Mitra, R., Buliung, R.N.: Built environment correlates of active school transportation: neighborhood and the modifiable areal unit problem. J. Transp. Geogr. 20, 51-61 (2012)

Nakanishi, H., Black, J.: Social sustainability issues and older adults' dependence on automobiles in lowdensity environments. Sustainability 7, 7289-7309 (2015)

Naumann, R.B., Dellinger, A.M., Anderson, M.E., Bonomi, A.E., Rivara, F.P., Thomson, R.S.: Preferred modes of travel among older adults: What factors affect the choice to walk instead of drive? J. Saf. Res. 40, 395-398 (2009)

Oxley, J., Whelan, M.: It cannot be all about safety: the benefits of prolonged mobility. Traffic Inj. Prev. 9, 367-378 (2008)

Páez, A., Scott, D., Potoglou, P., Kanarogou, P., Newbold, K.B.: Elderly mobility: demographic and spatial analysis of trip making in the Hamilton CMA, Canada. Urban Stud. 44, 123-146 (2007)

Perchoux, C., Chaix, B., Cummins, S., Kestens, Y.: Conceptualization and measurement of environmental exposure in epidemiology: accounting for activity space related to daily mobility. Health Place 21, 86-93 (2013)

Perchoux, C., Kestens, Y., Thomas, F., van Hulst, A., Thierry, B., Chaix, B.: Assessing patterns of spatial behavior in health studies: their socio-demographic determinants and associations with transportation modes (the RECORD Cohort Study). Soc. Sci. Med. 119, 64-73 (2014)

Pettersson, P., Schmöcker, J.D.: Active ageing in developing countries? Trip generation and tour complexity of older people in Metro Manila. J. Transp. Geogr. 18(5), 613-623 (2010)

Potoglou, D., Palacios, J.F., Feijóo, C.: An integrated latent variable and choice model to explore the role of privacy concern on stated behavioural intentions in e-commerce. J. Choice Model. 17, 10-27 (2015)

Ramezani, S., Pizzo, B., Deakin E.: Built environment versus personal traits: an application of integrated choice and latent variable model (ICLV) in understanding modal choice in Rome, Italy, In: Proceedings of the 14th International Conference on Computers in Urban Planning and Urban Management, Cambridge, MA (2015)

Ramezani, S., Pizzo, B., Deakin, E.: An integrated assessment of factors affecting modal choice, towards a better understanding of the causal effects of built environment. Transportation 45, 1351-1387 (2018a)

Ramezani, S., Pizzo, B., Deakin, E.: Determinants of sustainable mode choice in different socio-cultural contexts: a comparison of Rome and San Francisco. Int. J. Sustain. Transp. 12(9), 648-664 (2018b)

Saajanaho, M., Rantakokko, M., Portegijs, E., Törmäkangas, T., Eronen, J., Tsai, L., Jylhä, M., Rantanen, T.: Personal goals and changes in life-space mobility among older people. Prev. Med. 81, 163-167 (2015) 
Saajanaho, M., Viljanen, A., Read, S., Eronen, J., Kaprio, J., Jylhä, M., Rantanen, T.: Mobility limitation and changes in personal goals among older women. J. Gerontol. Ser. B Psychol. Sci. Soc. Sci. (2016). https://doi.org/10.1093/geronb/gbu094

Sallis, J.F., Cerin, E., Conway, T.L., et al.: Physical activity in relation to urban environments in 14 cities worldwide: a cross-sectional study. The Lancet 6736(16), 348 (2016)

Salmela-Aro, K.: Personal goals and well-being during critical life transitions: the four C's-Channeling, choice, co-agency and compensation. Adv. Life Course Res. 14(1-2), 63-73 (2009)

Salonen, M., Toivonen, T.: Modelling travel time in urban networks: comparable measures for private car and public transport. J. Transp. Geogr. 31, 143-153 (2013)

Schirmer, P.M., van Eggermond, M.A.B., Axhausen, K.W.: The role of location in residential location choice models: a review of literature. J. Transp. Land Use 7(2), 3-21 (2014)

Schmöker, J., Quddus, M.A., Noland, R.B., Bell, M.G.H.: Mode choice of older and disabled people: a case study of shopping trips in London. J. Transp. Geogr. 16, 257-267 (2008)

Schwanen, T., Mokhtarian, P.L.: What affects commute mode choice: Neighborhood physical structure or preferences toward neighborhoods? J. Transp. Geogr. 13, 83-99 (2005)

Schwanen, T., Dijst, M., Dieleman, F.M.: Leisure trips of senior citizens: determinants of modal choice. Tijdschr. voor Econ. Soc. Geogr. 92, 347-360 (2001)

Shao, F., Sui, Y., Yu, X., Sun, R.: Spatio-temporal travel patterns of elderly people-a comparative study based on buses usage in Qingdao, China. J. Transp. Geogr. 76, 178-190 (2019)

Su, F., Schmöcker, J., Bell, M.G.H.: Mode choice of older people before and after shopping. J. Transp. Land Use 2(1), 29-46 (2009)

Temme, D., Paulssen, M., Dannewald, T.: Incorporating latent variables into discrete choice models-a simultaneous estimation approach using SEM software. BuR-Bus. Res. 1(2), 220-237 (2008)

Theis, G.W.: Incorporating attitudes in airline itinerary choice: modelling the impact of elapsed time, $\mathrm{PhD}$ dissertation, Massachusetts Institute of Technology (2011)

United Nations: Report of the United Nations Second World Assembly on Aging (2002). http://www.un.org/ swaa2002/documents.htm. Accessed September 2017.

Van den Berg, P., Arentze, T., Timmermans, H.: Estimating social travel demand of senior citizens in the Netherlands. J. Transp. Geogr. 19, 323-331 (2011)

Vij, A., Walker, J.: Integrated choice and latent variable models: The holy grail or not? In: Paper Presented at the 13th International Conference of the International Association for Travel Behavior Research, Toronto (2012)

Vij, A., Walker, J.: How, when and why integrated choice and latent variable models are latently useful. Transp. Res. Part B 90, 192-217 (2016)

Wang, J., Kwan, M.-P., Chai, Y.: An innovative context-based crystal-growth activity space method for environmental exposure assessment: a study using GIS and GPS trajectory data collected in Chicago. Int. J. Environ. Res. Public Health 15(4), 703 (2018)

Wei, Q., She, J., Zhang, S., Ma, J.: Using individual GPS trajectories to explore foodscape exposure: a case study in Beijing metropolitan area. Int. J. Environ. Res. Public Health 15(3), 405 (2018)

Publisher's Note Springer Nature remains neutral with regard to jurisdictional claims in published maps and institutional affiliations.

Samira Ramezani works as a postdoctoral researcher with the Spatial Planning and Transportation Engineering research group of the Department of Built Environment of Aalto University, Finland. She holds a $\mathrm{PhD}$ in Urban and Regional Planning. Her research interests lie in the general areas of human-environment interactions, sustainable urban mobility and travel behavior, land use-transport interaction, and integrated land use and transport planning and policymaking.

Tiina Laatikainen is a postdoctoral researcher at the department of Built Environment of Aalto University, Finland. Her research interests mainly include health promoting aspects of the built environment and the human-environment interactions primarily from the active living perspective.

Kamyar Hasanzadeh is a post-doctoral researcher at the department of Built Environment of Aalto University, Finland. He has a background in Geoinformatics engineering and he has completed his doctoral studies in Land-use Planning and Transportation Engineering. Kamyar Hasanzadeh's research is mainly focused on developing innovative geospatial methods and tools for modeling individual behaviors, and using them to study the contextual effects of built environment on people. 
Marketta Kyttä works as a professor of Land Use Planning in Aalto University. She has her background in environmental psychology and participatory planning. Her research covers various topics: child-, and humanfriendly environments, environments that promote wellbeing and health, perceived safety and new methods for public participation. Currently, her multidisciplinary research team concentrates on the placebased person-environment research with public participation GIS methodology. 